\title{
GamAPI - Uma API para Gamificação
}

\section{GamAPI - A Gamification API}

JOSE LUIZ VILAS BOAS Universidade Estadual de Londrina (UEL)

MURILO AUGUSTO L. TEIXEIRA Universidade Estadual de Londrina (UEL)

EDUARDO FILGUEIRAS DAMACENO Universidade Tecnológica Federal do Paraná (UTFPR)

JACQUES DUÍLIO BRANCHER Universidade Estadual de Londrina (UEL)

Resumo: A Gamificação baseia-se na inserção elementos de jogo em um ambiente fora do seu escopo, com o objetivo de melhorar o engajamento e comprometimento de um indivíduo para a realização de uma tarefa. Para alcançar tal objetivo, a Gamificação trabalha com conceitos como: premiações; medalhas ou emblemas; progressão e um sistema de ranking. O objetivo deste trabalho é a criação de uma API que proporcione uma opção para implementar os conceitos e os mecanismos de gerenciamento de Gamificação, sem a necessidade de se ter uma estrutura própria e que conduza o aluno a uma experiência prazerosa na educação. Os resultados obtidos apontaram que, a API auxiliou no aumento da motivação dos alunos e os conduziu a este estado de imersão, contribuindo para o processo educacional.

Palavras-chave: Gamificação. Gamificação no Ensino. Engajamento.

Abstract: The Gamification is based on inserting game elements in an environment outside its scope, aiming at improving the users' engagement and motivation to perform a task. In order to achieve this goal, Gamification contains concepts as rewards, medals or badges, progression and ranking system. The aim of this work is the creation of an API that provides an option to implement the concepts and mechanisms of Gamification management, without the need to have a structure and leading the student to a pleasurable experience in education. The results obtained indicated that, the API has assisted in increasing the motivation of students and led them to this immersion state, contributing to the educational process.

Keywords: Gamification. Gamification in Teaching. Engagement. 


\section{Introdução}

A carência na motivação dos alunos no processo de ensino e aprendizagem tem-se tornado um problema para as escolas e professores (DOMÍNGUEZ et al., 2013). Segundo DICHEV et al. (2014) as estratégias didáticas utilizadas não conseguem estimular os alunos nas tarefas que Ihe são atribuídas, pois não estão em sintonia com a maneira como o aluno de apropria do conhecimento atualmente.

Ao se deparar com tal realidade, fica claro que os jovens de hoje, possuem uma visão completamente diferente de outrora (ALVES, 2014). Graças aos avanços tecnológicos, desenvolveram métodos alternativos para retenção de conhecimento, quebrando a premissa préestabelecida de que o processo de aprendizagem é enfadonho (PRENSKY, 2012). Estes métodos consideram a apresentação lúdica e a caracterização do espaço da aprendizagem para que os educadores consigam manter a atenção e o engajamento do indivíduo, no conteúdo ministrado.

Ademais, percebe-se que a motivação dos alunos e o seu envolvimento em sala de aula depende diretamente da atenção e do interesse provocado pelo professor, daí a abordagem que mais logra sucesso é a Gamificação (IOSUP; EPEMA, 2014). Desta forma os processos de Gamificação vêm emergindo consideravelmente nos últimos 5 anos (BRAZIL; BARUQUE, 2015), seja na indústria, na academia e no ensino propriamente relacionado.

Há formas de classificar os processos de Gamificação e que também a compõem, como por exemplo: Mecânica, Dinâmica e Estética. Estes transmitem emoções e inserem interações (ZICHERMANN; CUNNINGHAM, 2011) com o uso de elementos de jogo como premiações, emblemas, feedback e um sistema de ranking em ambientes de aprendizagem para aumentar a motivação dos alunos nas realizações de suas atividades (ROBSON et al., 2015).

A tecnologia advém para uma facilitação destes processos (ROBSON et al., 2015), todavia são encontradas limitações que certas ferramentas, como por exemplo, a não abordagem de todos estes recursos em uma única aplicação, extensão a diversos temas ou áreas, comunicação com outros sites e ferramentas e recursos de análise estatística. Esse último fornece uma gerência de informações que bem trabalhadas, devem ser capazes de criar meios do educador, melhorar sua metodologia de ensino para alcançar a imersão desejada, a fim de proporcionar uma experiência prazerosa no processo de ensino aprendizagem.

Neste sentido este artigo aborda uma estratégia que contribui ao disponibilizar para a comunidade acadêmica e geral, a opção de implementar os processos citados, com o uso de uma Ferramenta de Software, denominada GamAPI. As informações obtidas por meio da experimentação apontou que a ferramenta motivou os usuários na realização de suas atividades.

O trabalho está dividido de forma a dar o entendimento dos fundamentos necessários para a compreensão de Gamificação, seguido de uma análise bibliométrica dos trabalhos publicados entre os anos de 2012 a 2016. Complementando-se pela apresentação da pesquisa de campo realizada com os alunos de Engenharia da Computação da Universidade Tecnológica Federal do Paraná, campus Cornélio Procópio. Ao final são evidenciadas as contribuições para o estado da arte bem como os trabalhos futuros sobre o tema abordado.

\section{Gamificação}

A Gamificação é uma subárea da Game-Based Learning, definida como a utilização de mecânica de jogos, dinâmicas e estéticas em um ambiente que não seja o próprio jogo, a fim de engajar, motivar, treinar ou modificar o comportamento de um indivíduo (ZICHERMANN; CUNNINGHAM, 2011).

Esta definição de Gamificação contrasta outros conceitos de jogo, como os baseados em Serious Games (Jogos Sérios), cuja principal função é o aprendizado, deixando em segundo plano a diversão (MÜLLER et al., 2015) e Playful Interactions (Interações Lúdicas), que foca na utilização de brincadeiras para fins educacionais (DETERDING; DIXON, 2011). De acordo com DETERDING e DIXON (2011), a Gamificação situa-se dentro dos elementos de jogos digitais, possuindo, porém, algo a mais que somente a diversão. ZICHERMANN e CUNNINGHAM (2011) definem os seguintes elementos que compõem esta prática: Pontos, Níveis, Conquistas, Badges ou Emblemas, Leaderboard ou Ranking e o Feedback.

Os Pontos permitem quantificar o desempenho do aluno (IOSUP; EPEMA, 2014), auxiliando o usuário a estabelecer metas e objetivos. Os Níveis, assim como os Pontos, transmitem o 
progresso do jogador dentro do sistema (MÜLLER et al., 2015), estes determinam o grau de competência que uma pessoa possui dentro do ambiente (IOSUP; EPEMA, 2014).

As Conquistas são recompensas dadas quando um indivíduo alcança determinada pontuação (ELEFTHERIA et al., 2013), e podem estar associadas a um Emblema. Os Emblemas, tal quais as Conquistas e os Níveis, também são uma forma de transmitir o status, pois são um meio visual de representar as vitórias alcançadas (IOSUP; EPEMA, 2014). Para HAKULINEN e AUVINEN (2014), estes Emblemas podem ser usados como desafios extras, a fim de direcionar o usuário a um comportamento desejado.

Os Rankings ou Leaderboards são usados para expor os ganhos do usuário perante a comunidade a qual pertence, concebendo um meio de transmitir um incentivo social. Para ROBSON et al. (2015) é uma maneira de influenciar outros indivíduos que não estão no topo do ranking, a almejar essa posição despertando o espírito competitivo.

O Feedback tem uma função fundamental em um ambiente gamificado (ALVES, 2014) uma vez que auxilia a manter o usuário devidamente informado sobre o que está acontecendo no sistema. Caso seja mal trabalhado, não retornando em tempo real o resultado das suas ações sobre as mecânicas, tentando dirimir suas dúvidas à medida que acontecem, pode resultar em um distanciamento, acarretando no fracasso do ambiente (AMIR; RALPH, 2014).

A Dinâmica é a interação do usuário com o ambiente por meio das Mecânicas (ZICHERMANN; CUNNINGHAM, 2011). Uma Dinâmica envolvente é capaz de impulsionar um sistema gamificado (AMIR; RALPH, 2014), e, ao contrário das Mecânicas, que são definidas pelos designers, aquelas são fruto da ação dos jogadores sobre as regras (ROBSON et al., 2015). Já a Estética é a emoção que os jogadores estão sentindo durante a interação com o ambiente (ZICHERMANN; CUNNINGHAM, 2011) e, necessita ser positiva.

Verifica-se, portanto, que o uso destes elementos de jogos gera benefícios, tais como: maiores níveis de produtividade e de realizações individuais, e níveis de motivação elevados. Dessa forma, é perceptível que a utilização deste processo influencia positivamente a questão motivacional que subjaz.

\section{Trabalhos Correlatos}

Esta seção dedica-se a descrever os trabalhos desenvolvidos na área da Gamificação. Nesta cita-se as ferramentas que foram desenvolvidas para promover o engajamento dos indivíduos: ambientes, sites, plugins, API's e frameworks nas mais diversas tecnologias, voltados para área empresarial, educacional e outras.

A Generic Platform for Enterprise Gamification de HERZIG et al. (2012) foi desenvolvida para inserir a Gamificação em um ambiente empresarial, dentro de um ERP, para estimular os funcionários a utilizarem o sistema. Para alcançar este objetivo ele desenvolveu um protótipo de um ambiente estruturado em serviços. A plataforma contém um módulo chamado BRMS, que responde pelo cadastro das mecânicas de jogos; o Analytics, que proporciona uma análise dos dados gravados e a monitoração da participação dos usuários; o Message Broker, que fornece a saída gerada pelos métodos programados no serviço e que permite ao usuário captar a saída através de eventos no front e tratar como for conveniente.

DOMÍNGUEZ et al. (2013) desenvolveram uma API para uso em conjunto com a plataforma E-Learning chamado Blackboard. Com o objetivo de tornar a ferramenta mais simples os autores optaram por trabalhar com apenas quatro níveis, a saber: Cobre, Prata, Ouro e Platina. Eles são visualizados em forma de figuras, imitando o esquema de troféus e/ou medalhas.

IBANEZ et al. (2014) elaboraram um estudo para analisar a eficácia da utilização da Gamificação e melhorar o engajamento de estudantes em programação C no curso de Ciência da Computação. O objetivo principal da pesquisa foi explorar o impacto dela sobre o engajamento dos estudantes, bem como a compreensão, análise do envolvimento dos alunos e o desempenho acadêmico.

Para aplicar esta pesquisa foi desenvolvida uma plataforma Q-Learning, com os elementos básicos da mecânica de jogo. Os estudantes precisavam inserir e avaliar questões relacionadas à linguagem de programação $C$, consequentemente, aumentando seus níveis de especialização.

Em linhas gerais, é fundamental ainda descrever a plataforma e API CAPTAIN UP (2016), que assim como a API da presente pesquisa, possui o cadastro das mecânicas, o gerenciamento de informações, além de ligação com outras tecnologias baseadas JavaScript. 
O módulo de gerenciamento permite ao administrador cadastrar Pontos, Emblemas, Níveis, e Recompensas para incentivar os usuários a executar ações desejáveis com o uso de mensagens e ranking. Também possui instrumentos que permitem gerenciar as atividades do usuário. No entanto, é importante frisar que esta ferramenta não é gratuita, e tem seu foco em aplicações empresariais, podendo ser estendida a outras áreas. A versão de teste permite ao usuário certo número de ações, como a criação de até dez Conquistas e oito Níveis.

\section{Desenvolvimento do GamAPI}

Os métodos de Gamificação utilizados para compor a $A P I$ foram elaborados a partir de estudos teóricos de autores como ZICHERMANN e CUNNINGHAM (2011) e IOSUP e EPEMA (2014). Estes apresentam o uso de recursos tecnológicos e o gerenciamento do processo de aprendizagem baseado na dinâmica e mecânica de jogos. A Figura 1 apresenta um esquema da relação entre a $A P I$ e os diversos perfis de usuários e ambientes.

Analisando a Figura 1, a esquerda tem-se os recursos do Servidor GamAPI como: Banco de Dados, Sistema Gerencial representado pela Página de Admin e os Métodos do Web Service. Ao utilizar um serviço, busca-se uma customização e integração com qualquer ambiente, outros sites e ferramentas (LECHETA, 2010). Já a Página de Admin, permite aos usuários administradores criar Comunidades dentro do sistema, inserir Emblemas, Pontos, Conquistas bem como gerenciar as informações e monitorar as ações dos indivíduos pertencentes a sua comunidade.

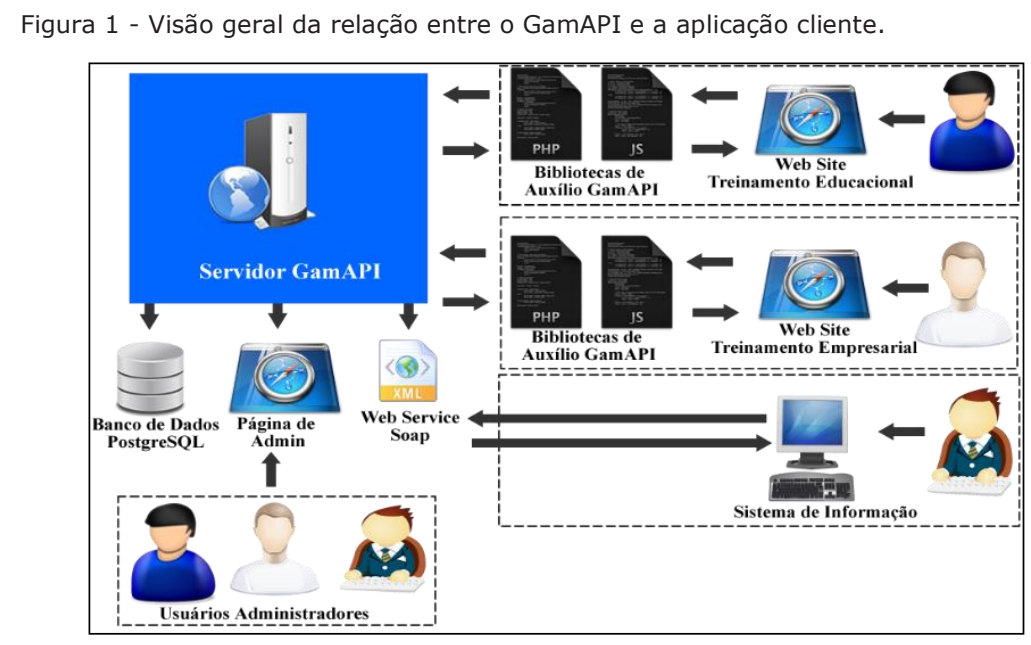

Fonte: Elaborada pelo Autor.

Para promover a usabilidade do sistema por diversos desenvolvedores, e tendo como foco os menos experientes, foi desenvolvido um conjunto de Bibliotecas de Auxílio, em PHP e JavaScript. Elas funcionam como um agente intermediário com o Web Service. Sua utilização permite que haja uma maneira padrão de acesso ao serviço por parte das linguagens voltadas para web, além de encapsular toda a programação, restando apenas ao usuário fornecer os parâmetros corretos. As bibliotecas e a documentação para a implementação no site podem ser adquiridas por meio de acesso ao endereço eletrônico http://www.gamapi.com.br.

\subsection{Módulo Administrador do API}

O Módulo Administrador foi desenvolvido na linguagem PHP versão 5. Essa variante permite que seja feita a inserção de todas as regras que serão usadas na execução do processo de Gamificação dentro de um site ou sistema, tais como: o cadastro de usuários, o cadastro da comunidade, ou a escolha de qual se deseja participar, cadastro de níveis, de conquistas e monitoramento da ação do usuário sobre as mecânicas de jogos. A demonstração dos recursos disponibilizados pelo GamAPI está presente na seção de Método de Pesquisa, bem como no manual disponível no site da $A P I$. 


\section{Método de Pesquisa}

Um quasi-experimento foi realizado para a validação do GamAPI. A amostragem selecionada foram 10 alunos do curso de Graduação em Engenharia da Computação da Universidade Tecnológica Federal do Paraná - Campus Cornélio Procópio. O objetivo foi verificar se a API causou o efeito esperado, validando a proposta deste trabalho.

Com base no objetivo apresentado surgiram as hipóteses $\mathrm{H} 1-0, \mathrm{H} 1-1$ e H2, que buscam responder à questão norteadora deste trabalho: "A aplicação do GamAPI é um fator motivador para os usuários?"

- H1-0: A utilização do GamAPI em conjunto com as técnicas de Gamificação em ambientes de treinamento não influenciam na motivação dos usuários.

- H1-1: A utilização do GamAPI em conjunto com as técnicas de Gamificação em ambientes de treinamento influenciam na motivação dos usuários.

- H2: As funcionalidades do GamAPI atendem aos requisitos de interação com os usuários.

Para responder aos questionamentos hipotéticos, houveram quatro momentos para a inserção da ferramenta, a saber: cadastro das questões no Ambiente Virtual de Treinamento Gamificado (AVTGam); cadastro das mecânicas no GamAPI; utilização do GamAPI no site de ensino, e o processamento dos dados.

\subsection{Preparações do ambiente no AVTGam}

Para comprovar a eficiência da $A P I$, ela foi inserida em um ambiente de treinamento real, 0 AVTGam. Esse ambiente foi desenvolvido como parte da pesquisa, objetivando comprovar a eficiência do GamAPI, bem como disponibilizar uma ferramenta de aprendizagem para a comunidade acadêmica. A figura 2 exibe a tela principal do AVTGam.

Figura 2 - Tela principal do AVTGam.

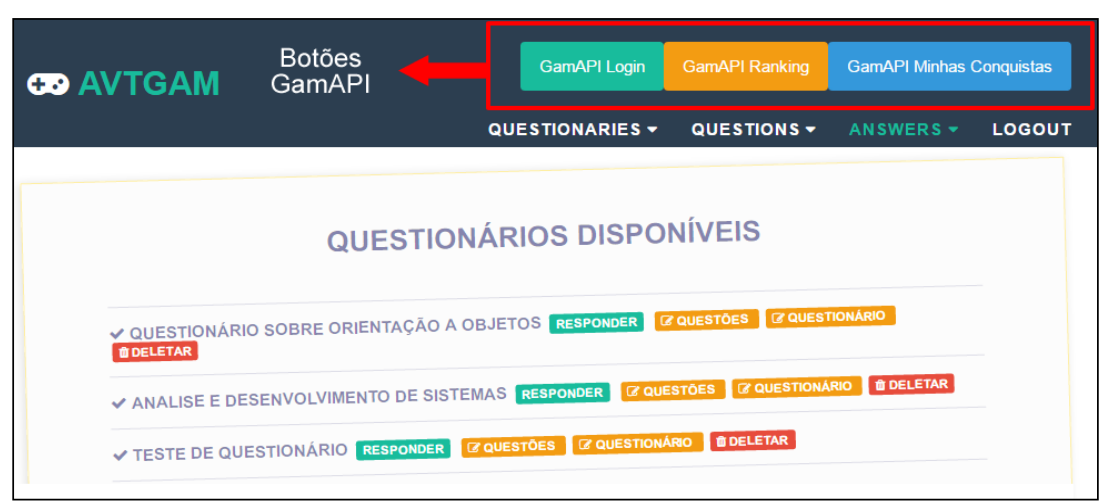

Fonte: Elaborado pelo Autor.

Na formatação do ambiente foi configurado um questionário com exercícios referentes aos conceitos de Orientação a Objetos, verificável na Figura 2. Para utilizá-lo, o docente responsável acessou ao sistema no endereço http://www.gamapi.com.br/quest, efetuou o cadastro e inseriu um total de 16 questões, todas de múltipla escolha.

\subsection{Cadastros das Mecânicas no GamAPI}

O professor criou uma comunidade no GamAPI denominada IF69N-C101, com o tema Tópicos em Engenharia de Software. Nela foram cadastradas inicialmente 4 conquistas e 2 níveis. Os passos necessários para o cadastro das mecânicas estão disponíveis na documentação. O resultado final do cadastro das mecânicas e o painel de administração podem ser observados na Figura 3. 
Figura 3. Painel de Administração da Comunidade.

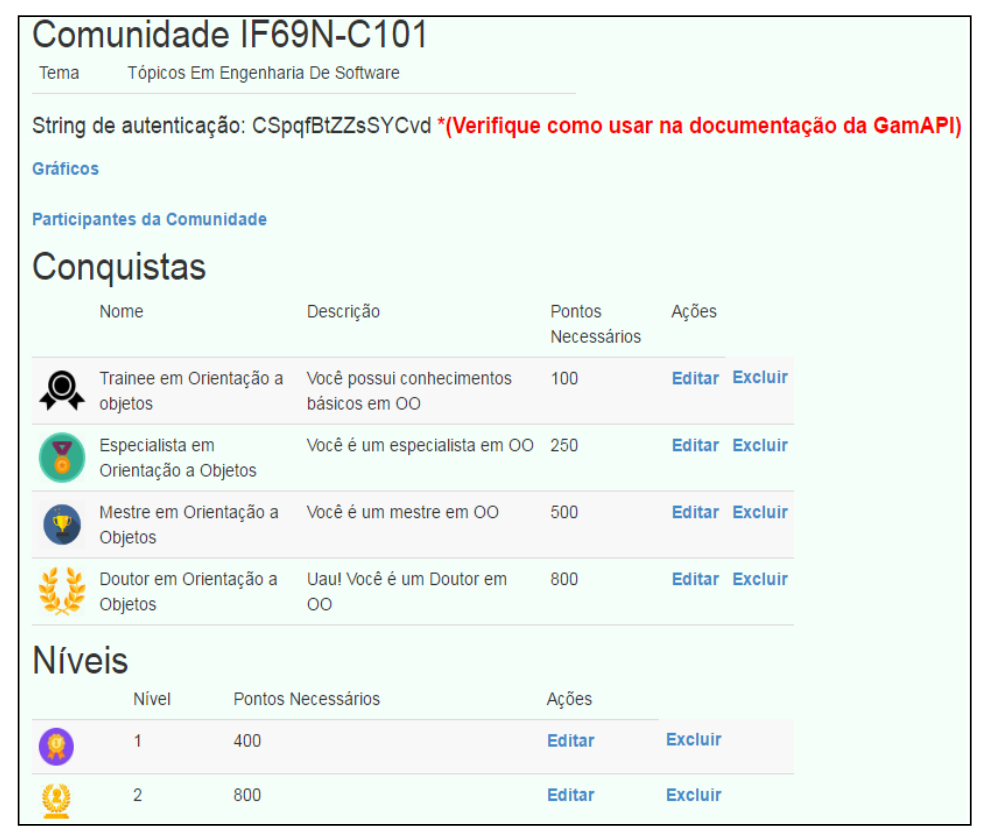

Fonte: Elaborada pelo Autor.

Na Figura 3 constam as conquistas cadastradas pelo professor: Trainee em Orientação a Objetos, com 100 pontos necessários para o seu desbloqueio; Especialista em Orientação a Objetos, com 250 pontos; Mestre em Orientação a Objetos, com 500 pontos e, Doutor em Orientação a Objetivos, com 800 pontos. Ainda na Figura 3 estão visíveis os níveis cadastrados, com seus respectivos pontos necessários para a liberação, sendo 400 e 800 , respectivamente.

A API também fornece outros recursos, tais como: o gráfico comparativo de progressão entre os indivíduos, que dispõe informações relacionadas às conquistas cadastradas no sistema, e o que cada um conseguiu desbloquear; um resumo que exibe o que os indivíduos conquistaram até o momento, como os níveis alcançados, a quantidade de pontos adquiridos e as conquistas desbloqueadas. Também fornece os acessos dos usuários no dia, o tempo que cada interagiu com o sistema, os dias da semana e os itens conquistados neste período.

\subsection{Utilização do GamAPI}

Esta seção dedica-se a demonstrar a interatividade do GamAPI em um ambiente de ensino. No início, cada aluno do curso de Engenharia da Computação foi apresentado aos ambientes, tomando conhecimento de detalhes dos procedimentos para o ingresso tanto na $A P I$ como no AVTGam. Para acessar o GamAPI é necessário que o usuário utilizador cadastre-se, da mesma forma como faz o usuário administrador, selecionando depois em qual comunidade deseja ingressar (Figura 4).

Figura 4. Participar da Comunidade no GamAPI. 


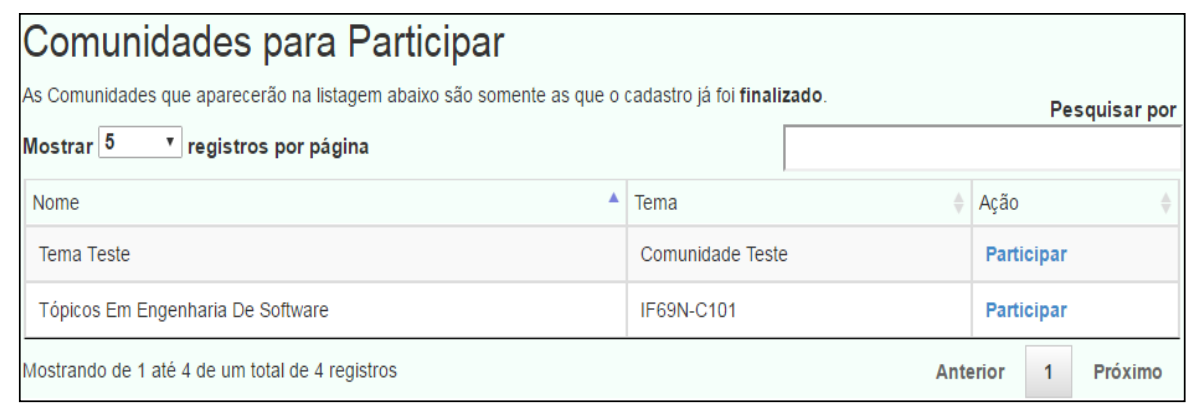

Fonte: Elaborada pelo Autor.

Após escolher a comunidade (Figura 4), o usuário está apto a utilizar o AVTGam com todos os recursos de Gamificação da GamAPI. Para exemplificar a interação do processo, a Figura 5 mostra, por meio do item $A$, as conquistas do usuário, e no item $B$, o ranking de usuários. Figura 5. Exemplos de recursos de feedback para o usuário

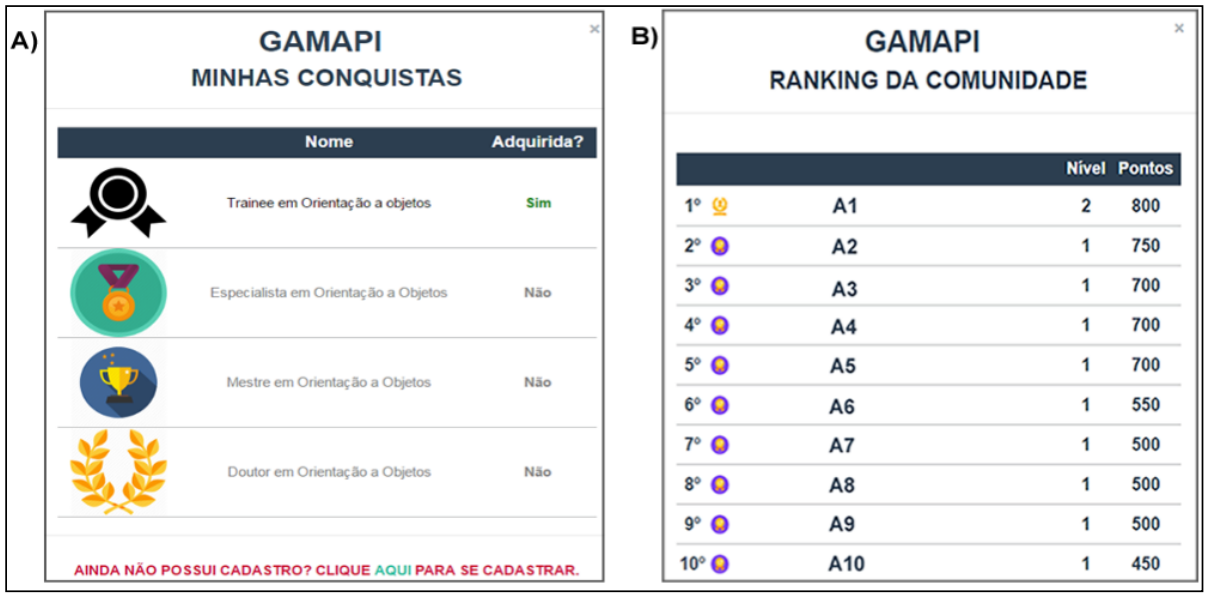

Fonte: Elaborado pelo Autor.

Na figura 5A é possível visualizar as conquistas do usuário autenticado, bem como aquelas que ainda não alcançou. A progressão ajuda a manter o usuário no sistema, pois fornece meios para que ele possa estabelecer seus objetivos, e, deste modo, mantém o usuário motivado, garantindo o sucesso do sistema (MORRISON; DISALVO, 2014).

A figura 5B exibe o ranking dos usuários da comunidade IF69N-C101 por pontuação. Também são apresentados seus níveis e a badge correspondente a estes. Esse tipo de mecânica, quando inserida dentro de uma rede social, neste caso específico representada pelo item Comunidade, transmite status, desperta o espírito competitivo e permite aos usuários analisar suas próprias performances (IOSUP; EPEMA, 2014).

Outros recursos do GamAPI também estão presentes no site, tais como: mensagens informativas sobre os pontos ganhos a cada submissão correta, as conquistas adquiridas e o nível ao qual usuário eleva quando alcança a pontuação inserida no sistema gerencial do GamAPI. Esse feedback imediato e em tempo real propicia a percepção de imersão no sistema (ELEFTHERIA et al., 2013). Segundo AKPOLAT e SLANY (2014), a atualização contínua motiva o usuário, trazendo a ele uma sensação de transparência, fator que auxilia para que todos se mantenham no ambiente, além disso, possibilita quantificar o desempenho do aluno (IOSUP; EPEMA, 2014).

\subsection{Apresentação dos Resultados e Discussões}

Esta seção dedica-se a descrever os resultados obtidos por meio da utilização do método de estatística descritiva, utilizado para processar os dados. Tal método utiliza de ferramentas como tabelas, gráficos e cálculos estatísticos para sumarizar os resultados (AKANIME; YAMAMOTO, 2009). A partir dessa metodologia, foram criados gráficos que sintetizam visivelmente as opiniões dos alunos, adquiridas por meio do Survey, disponíveis em: 
https://www.dropbox.com/s/hdzzemhcoe1g3yq/respostas.rar?dl=0. O primeiro gráfico (Figura 6) expõe as opiniões dos alunos que almejaram melhorar sua colocação ao visualizar o ranking.

Figura 6. Gráfico do percentual de alunos que almejaram o topo do ranking

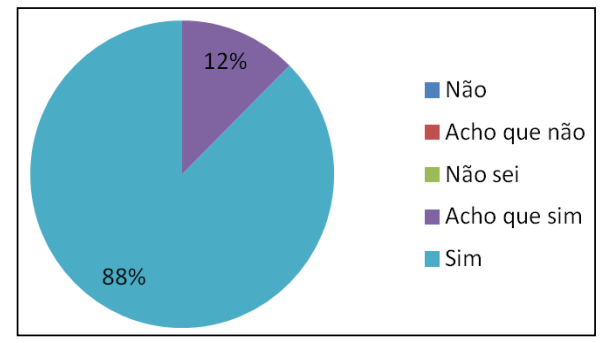

Fonte: Elaborada pelo Autor.

Com base nas informações do gráfico da Figura 6, praticamente $100 \%$ dos alunos desejaram melhorar sua colocação após a visualização do ranking. Ainda que 1 aluno (12\%), não tenha sinalizando veementemente o desejo de elevar seu nível, demonstrou ao menos o interesse, em razão ter assinalado a opção Acho que sim. O gráfico da Figura 7 traz o questionamento a respeito da percepção do indivíduo no que diz respeito à evolução dos seus pontos. Os alunos foram questionados se perceberam a evolução dos seus pontos e conquistas até o final das atividades.

Figura 7. Gráfico do percentual de alunos que constataram a evolução dos seus pontos e conquistas.

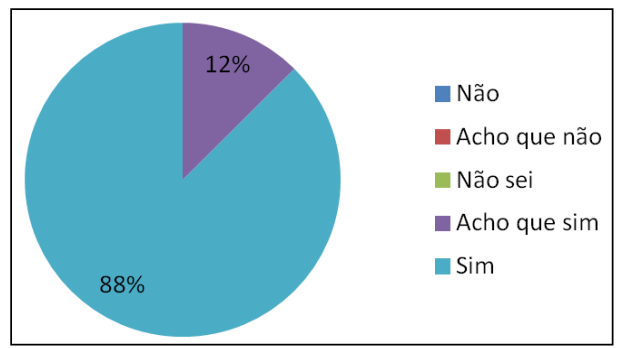

Fonte: Elaborada pelo Autor.

O gráfico da Figura 7 demonstra que praticamente todos os envolvidos visualizaram a evolução dos seus pontos, mesmo que uma minoria não tenha acompanhado tal dado. O último gráfico (Figura 8) demonstra a avaliação dos participantes em relação às respostas do sistema, denotando se houve interação, ou seja, se fora apresentada a pontuação em tempo hábil.

Figura 8. Gráfico do percentual de alunos consideram o feedback do GamAPI em tempo hábil.

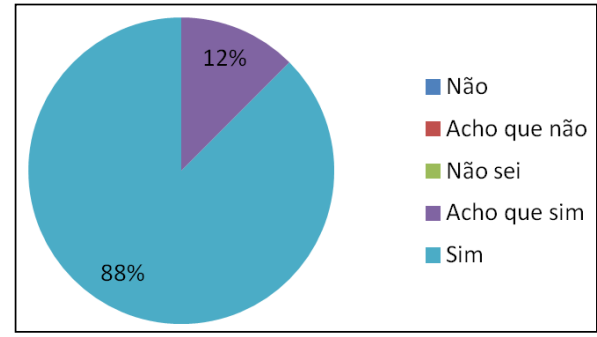

Fonte: Elaborada pelo Autor.

Ao analisar o gráfico (Figura 8), nota-se que para a maioria dos alunos o sistema apresentou as mensagens de feedback em tempo hábil. $\mathrm{E}$, mesmo que um aluno tenha optado pela resposta "Acho que Sim", esta não contradiz o "Sim", fato o que garante 100\% de afirmação. Caso o aluno não concordasse, havia a opção de assinalar "Não", "Acho que não", ou mesmo "Não sei".

Os dados adquiridos a partir da análise dos resultados do questionário corroboram a efetividade do uso da GamAPI, pois os alunos interagiram positivamente com as funcionalidades da API. Tal consequência é visível nos gráficos das Figuras 6, 7 e 8, o que valida à hipótese $\mathrm{H} 2$. Levando em conta tais circunstâncias, houve a demonstração, durante aplicação do teste, do 
anseio pelas posições superiores do ranking, observadas na Figura 6. É possível afirmar ainda que fora notada certa euforia, por parte dos alunos, ao conseguirem desbloquear as conquistas ou evoluir de nível, o que refuta a hipótese nula $\mathrm{H} 1-0$.

Estas observações, juntamente com os dados obtidos, compõem indícios positivos de que a $A P I$ tem seu propósito válido, o que reafirma as hipóteses $\mathrm{H} 1-1$ e $\mathrm{H} 2$. Neste caso, ela funcionou corretamente, promovendo a interação com o usuário em tempo real, e, em conjunto com as técnicas de Gamificação, influenciou positivamente os alunos na resolução de suas atividades.

\section{Conclusões e Trabalhos Futuros}

Este estudo teve como enfoque principal o desenvolvimento de uma API para implementar o processo de Gamificação em aplicações de ensino/treinamento, com base nos sistemas de pontos, conquistas e placares. Um fator importante nesse emprego relaciona-se ao fato de que todo o processamento é realizado no próprio servidor, baseando-se na tecnologia de Web Services.

Embora a amostragem empregada no quasi-experimento tenha sido restrita, os alunos interagiram positivamente com o GamAPI, demonstrando, durante aplicação, bom desempenho ao desbloquear as conquistas e/ou evoluir de nível, conforme mencionado.

Como trabalho futuro pretende-se pôr em prática o uso da GamAPI, em conjunto com o AVTGam, nas disciplinas de Estrutura de Dados e Laboratório de Informática, pertencentes à graduação em Análise e Desenvolvimento de Sistemas, efetuando o monitoramento das atividades por um semestre.

\section{Referências}

AKANIME, C. T.; YAMAMOTO, R. K. Estudo dirigido de estatística descritiva. $2^{a}$ Ed. São Paulo. Editora Érica, 2009.

AKPOLAT, B. S.; SLANY, W. Enhancing software engineering student team engagement in a high-intensity extreme programming course using gamification. 2014 IEEE 27th Conference on Software Engineering Education and Training, CSEE and T 2014 - Proceedings, p. 149-153, 2014.

ALVES, F. Gamification: Como criar experiências de aprendizagem engajadoras. Um guia completo: do conceito à pratica. DVS Editora, 2014.

AMIR, B.; RALPH, P. Proposing a theory of gamification effectiveness. Companion Proceedings of the 36th International Conference on Software Engineering - ICSE Companion 2014, p. 626-627, 2014.

BRAZIL, A. L.; BARUQUE, L. B. Gamificação Aplicada na Graduação em Jogos Digitais. Anais do XXVI Simpósio Brasileiro de Informática na Educação (SBIE 2015), n. Sbie, p. 677-686, 2015.

CAPTAIN UP. Disponível em: <https://captainup.com/>. Acesso em 25/10/2016.

DETERDING, S.; DIXON, D. From Game Design Elements to Gamefulness: Defining "Gamification ". Proceedings from MindTrek '11. Tampere, Finland: ACM, p. 9-15, 2011.

DICHEVA, D. et al. Gamification in education: A systematic mapping study. Educational Technology and Society, v. 18, n. 3, p. 75-88, 2015.

DOMÍNGUEZ, A. et al. Gamifying learning experiences: Practical implications and outcomes. Computers and Education, v. 63, p. 380-392, 2013.

ELEFTHERIA, C. A. et al. An innovative augmented reality educational platform using Gamification to enhance lifelong learning and cultural education. IISA 2013 - 4th International Conference on Information, Intelligence, Systems and Applications, p. 258-263, 2013.

HAKULINEN, L.; AUVINEN, T. The effect of gamification on students with different achievement goal orientations. Proceedings - 2014 International Conference on Teaching and Learning in Computing and Engineering, LATICE 2014, p. 9-16, 2014. 
HERZIG, P.; AMELING, M.; SCHILL, A. (2012). A Generic Platform for Enterprise Gamification. Joint Working IEEE/IFIP Conference on Software Architecture and European Conference on Software Architecture, 219-223, 2012.

IBANEZ, M.-B.; DI-SERIO, A.; DELGADO-KLOOS, C. Gamification for Engaging Computer Science Students in Learning Activities: A Case Study. IEEE Transactions on Learning Technologies, v. 7, n. 3, p. 291-301, 2014.

IOSUP, A.; EPEMA, D. An experience report on using gamification in technical higher education. Proceedings of the 45th ACM technical symposium on Computer science education - SIGCSE '14, n. 2008, p. 27-32, 2014.

LECHETA, R. R. Google Android : Aprenda a criar aplicações para dispositivos móveis com o Android SDK, $2^{a}$ Ed. São Paulo. Novatec, 2010.

MORRISON, B. B.; DISALVO, B. Khan academy gamifies computer science. Proceedings of the 45th ACM technical symposium on Computer science education - SIGCSE '14, n. September 2015, p. 39-44, 2014.

MÜLLER, B. C.; REISE, C.; SELIGER, G. Gamification in Factory Management Education - A Case Study with Lego Mindstorms. Procedia CIRP, v. 26, n. Crc 1026, p. 121-126, 2015.

PRENSKY, M. Aprendizagem baseada em jogos digitais. São Paulo: Editora Senac, São Paulo, 2012.

ROBSON, K. et al. Is it all a game? Understanding the principles of gamification. Business Horizons, v. 58, n. 4, p. 411-420, 2015.

ZICHERMANN, G.; CUNNINGHAM, C. Gamification by Design: Implementing Game Mechanics in Web and Mobile Apps. O'Reilly Media; 1 edition, 2011.

Submetido para avaliação em 8 de dezembro de 2016 Aprovado para publicação em 22 de março de 2017

\section{Jose Luiz Vilas Boas}

Departamento de Computação - Universidade Estadual de Londrina - UEL, Brasil, joseluizvilasboas@gmail.com

\section{Murilo Augusto Lopes Teixeira}

Departamento de Computação - Universidade Estadual de Londrina - UEL, Brasil, murilo.lopest@gmail.com

\section{Eduardo Filgueiras Damaceno}

Universidade Tecnológica Federal do Paraná - UTFPR, Brasil, damasceno@utfpr.edu.br

\section{Jacques Duílio Brancher}

Departamento de Computação - Universidade Estadual de Londrina - UEL, Brasil, jacques@uel.br 